\title{
Host-guest interactions involving cyclodextrins: useful complementary insights achieved by polarimetry
}

\author{
Paolo Lo Meo, ${ }^{*}$ Francesca D'Anna, Serena Riela, Michelangelo Gruttadauria \\ and Renato Noto* \\ Dipartimento di Chimica Organica 'E. Paternò', Università degli Studi di Palermo, V.le delle Scienze, Parco d'Orleans II, \\ Pad. 17-90128 Palermo, Italy
}

Received 20 March 2007; revised 1 June 2007; accepted 21 June 2007

Available online 27 June 2007

Dedicated to Professor Domenico Spinelli on the occasion of his 75th birthday

\begin{abstract}
By means of simple polarimetry, we studied the binding abilities of native $\alpha$-, $\beta$-, and $\gamma$-cyclodextrins toward a group of suitably chosen model guests. We were able to get reliable estimations of the binding constants $K$, spread over a wide range (from 3.7 to $12,300 \mathrm{M}^{-1}$ ), allowing us to carry on interesting comparisons. A comprehensive discussion of polarimetric data, and in particular a detailed analysis of the variations $\Delta \Theta$ of molar optical activities consequent to inclusion, offered us the opportunity to get useful insights into the structure and dynamic behavior of host-guest complexes.

(C) 2007 Elsevier Ltd. All rights reserved.
\end{abstract}

\section{Introduction}

Owing to their ability to form inclusion complexes with a large number of suitably sized and structured organic guest molecules, ${ }^{1}$ native and chemically modified cyclodextrins (CDs) are materials of enormous interest. They are employed in widespread research and industrial applications, spanning from reaction microenvironments ${ }^{2}$ and enzyme modeling ${ }^{3}$ to separation technologies ${ }^{4}$ and to additives for pharmaceutical, cosmetic, and food industries. ${ }^{5}$ Studies on CDs are the object of an immense literature that is periodically reviewed. ${ }^{6}$ Under this perspective, a deep understanding of the microscopic features of the binding phenomenon, and of the factors affecting the thermodynamics of the inclusion process, is of fundamental importance.

Despite the large amount of both experimental and computational work carried out during the last decades, the intimate mechanisms implied in molecular and chiral recognition by CDs are still object of an intense debate and, up to now, cannot be considered satisfactorily understood. A thorough analysis of available data shows that inclusion is affected by different factors (hydrophobic and dipolar/ electrostatic interactions, hydrogen bond or other specific interactions, release of steric strain, solvent reorganization), often working in opposition to each other, and with no obvious hierarchy among them. ${ }^{7}$ All these effects exert their own

\footnotetext{
* Corresponding authors. Tel.: +39 091 596919; fax: +39091 596825; e-mail: rnoto@unipa.it
}

influence on both the $\Delta H^{\mathrm{o}}$ and $T \Delta S^{\mathrm{o}}$ parameters associated with the process. The intuitive idea that a stronger interaction should correspond with a more rigid inclusion complex, suggested the possibility of a $\Delta H^{\circ}-T \Delta S^{\mathrm{o}}$ 'isoequilibrium' compensation effect, ${ }^{8,9}$ a topic heavily debated until recently. $^{10-12}$ In general, the inclusion is mainly enthalpydriven when 'non-specific' effects (hydrophobic, van der Waals or dipolar interactions, solvation effects) prevail. ${ }^{9,12}$ However, when strong 'specific' interactions (such as multiple hydrogen bonding) occur, unfavorable entropy effects may overwhelm enthalpy contributions. ${ }^{11}$ Furthermore, on some occasions, the molecular recognition properties of CDs have not been found as effective as reasonably expected. For instance, enantioselectivities of native CDs toward simple chiral derivatives are modest, ${ }^{9,13}$ whereas significant improvements may be achieved in constrained systems. ${ }^{14}$ As a matter of fact, the possibility of an 'induced fit' effect for the CD host may allow the optimization of molecular interactions with both enantiomers of the chiral guest. ${ }^{15}$ Indeed, the dynamic behavior of CDs as flexible objects $^{16}$ is a topic that probably deserves more attention now than in the past: in fact, the old image of CDs as rigid and symmetric buckets has been progressively abandoned and should now be considered obsolete.

The need to investigate more deeply host-guest inclusion phenomena in general, and binding equilibria involving CDs in particular, has encouraged the search for new experimental methodologies and procedures, able to achieve more precise, reliable and detailed information, and more quickly. 


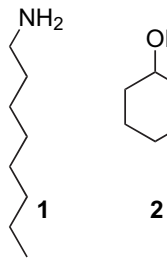<smiles>Oc1ccccc1</smiles><smiles>O=C(O)c1ccccc1</smiles><smiles>O=[N+]([O-])c1ccc(O)cc1</smiles>

8<smiles>O=[N+]([O-])c1ccc(N(CCO)CCO)cc1</smiles>

12<smiles>O=C(O)CNc1ccc([N+](=O)[O-])cc1</smiles>

13<smiles>O=C(O)C1CCCCC1</smiles>

3

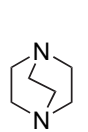

4

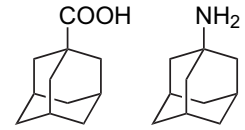

5

6<smiles>CN(CCO)c1ccc([N+](=O)[O-])cc1</smiles>

10

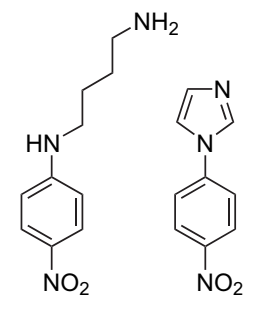

15<smiles>CC(Nc1ccc([N+](=O)[O-])cc1)C(=O)O</smiles>

$14-R$

Figure 1. Guests 1-16.

Current methods are based on the detection of a variation in any suitable physical/chemical property of the system upon inclusion. ${ }^{17}$ More common protocols, exploiting spectrometric (UV-vis, NMR, fluorescence, induced circular dichroism), chromatographic or calorimetric tools, are focused on the properties of the guest. However, we have recently shown that an interesting alternative approach to the problem may profitably exploit the properties of the host by means of polarimetry, as an experimental tool allowing to minimize the waste of time and materials needed. ${ }^{18}$ In the present paper we have extended our investigation, focusing not only on the methodological aspects of this technique but also on the overall interpretation of experimental data within a comprehensive framework. For these purposes, we evaluated the binding constants between native $\alpha-\mathbf{C D}, \beta-\mathbf{C D}$, and $\gamma-\mathbf{C D}$ and a set of suitable model guest molecules 1-16 (Fig. 1).

The chosen guests present significant variations in different molecular properties (steric bulk, conformational flexibility, hydrophobicity, polarizability, ability to act as hydrogen bond donor/acceptor). Most of them have an ionizable group, so measurements were performed using aqueous buffers at suitable $\mathrm{pH}$ values $(2.5,6.0,9.2,11.0)$. On the grounds of the literature reports, ${ }^{7 f}$ the guests were selected in such a way to show large variations in the binding affinities toward the $\mathbf{C D}$ hosts, i.e., in order to explore a range of $K$ values, which was as wide as possible.

\section{Results and discussion}

It is well known that polysaccharides owe their optical activity to their conformational behavior as well as to their

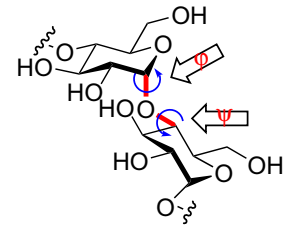

Figure 2. The glycosidic link double rotor.

intrinsic chirality. In particular, Rees ${ }^{19}$ investigated the contribution to the optical activities of oligosaccharides deriving from the 'linkage conformation', related to the (virtually) free rotation around the $\mathrm{C}(1)-\mathrm{O}-\mathrm{C}\left(4^{\prime}\right)$ glycosidic link double rotor, and defined as the pair of values assumed by the relevant dihedral angles $\varphi$ and $\psi$ (Fig. 2).

Our approach to the polarimetric study of host-guest interactions is based on the simple idea that the optical activity of a CD should significantly change upon guest inclusion, as a consequence of its conformational rearrangement and modified dynamic behavior, as well as of the micro-environmental effect of the guest itself. The latter contribution can be viewed as arising both from the polar effect of the guest on the electron distribution of the host molecule, and from an ICD effect on any guest chromophore. The topic of ICD in cyclodextrin complexes has been thoroughly discussed from a theoretical point of view, and some general rules have been assessed. ${ }^{20}$

Before proceeding with the examination of our results, a few words should be given about some features of our measurement procedure. As illustrated in Section 4, for a typical experiment a set of samples is prepared by mixing fixed amounts of a unique aqueous mother $\mathbf{C D}$ solution with varying micro-amounts of a concentrated stock solution of the guest dissolved in methanol (or in any other solvent miscible with water). Because the volume of organic solvent added to each sample (up to 4\%) is 'small' with respect to the volume of the aqueous solution, we may reasonably assume that the effect of the organic co-solvent on the actual value of the binding constant $K$ is negligible as compared to the experimental indeterminations. ${ }^{21}$ In other words, the effect of the organic co-solvent on the actual position of the binding equilibrium is assumed to be only that due to dilution. We can also assume that the actual volumes $V_{\mathrm{i}}$ of the samples follow the simple linear relationship Eq. 1:

$V_{\mathrm{i}}=V_{\mathrm{o}}\left(1+f v_{\mathrm{i}} / V_{\mathrm{o}}\right)$

where $v_{\mathrm{i}}$ is the micro-amount of guest solution added, $V_{\mathrm{o}}$ is the fixed volume of $\mathbf{C D}$ mother solution, and the coefficient $f$ simply accounts for the partial molar volume of the organic solvent in water. ${ }^{22}$ Under these conditions, in the most general case of the interaction of the $\mathbf{C D}$ with a chiral guest, it can be algebraically shown (see Supplementary data) that the observed optical rotation $\vartheta_{\mathrm{i}}$ of each sample follows the relationship Eq. 2 (see below), where $\vartheta_{\mathrm{o}}$ is the optical rotation of the mother $\mathbf{C D}$ solution, $G_{\mathrm{o}}$ is the analytical concentration of the guest mother solution, $\left[\Theta_{\mathrm{G}}\right]$ is the molar optical activity of the free guest, ${ }^{23}$ and $\Delta \Theta$ is the difference between the molar optical activity of the inclusion complex and the sum of the molar optical activities of the free host and guest. 
As we will illustrate in detail hereafter, the latter parameter plays a main role in the interpretation of our results. As a matter of fact, a careful analysis of its trends may provide useful complementary insights into the dynamic intimate life of the host-guest complexes. Finally, because we deal more commonly with achiral guests, Eq. 2 can be simplified as 3 (see below), which is the fitting equation already reported in our previous paper. Experimental data collected for the present work are summarized in Table 1. be profitably applied to the investigation of guests, which have been difficult to study otherwise. Moreover, and most importantly, this method allows to study under the same conditions guests having non-homogeneous characteristics, which are consequently hard to study by means of the same methodology, and the comparison of which has been made difficult previously because of the lack of methodologically homogeneous data. A quite large range of $K$ values could be explored (spanning from 3.7 to

$\vartheta_{\mathrm{i}}=\frac{\vartheta_{\mathrm{o}}+\left[\Theta_{\mathrm{G}}\right] G_{\mathrm{o}} \frac{v_{\mathrm{i}}}{V_{\mathrm{o}}}+\frac{\Delta \Theta}{2}\left(\mathbf{C D}_{\mathrm{o}}+G_{\mathrm{o}} \frac{v_{\mathrm{i}}}{V_{\mathrm{o}}}+\frac{1+f v_{\mathrm{i}} / V_{\mathrm{o}}}{K}-\sqrt{\left(\mathbf{C D}_{\mathrm{o}}+G_{\mathrm{o}} \frac{v_{\mathrm{i}}}{V_{\mathrm{o}}}+\frac{1+f v_{\mathrm{i}} / V_{\mathrm{o}}}{K}\right)^{2}-4 \mathbf{C D}_{\mathrm{o}} G_{\mathrm{o}} \frac{v_{\mathrm{i}}}{V_{\mathrm{o}}}}\right)}{1+f v_{\mathrm{i}} / V_{\mathrm{o}}}$

$\vartheta_{\mathrm{i}}=\frac{\vartheta_{\mathrm{o}}+\frac{\Delta \Theta}{2}\left(\mathbf{C D}_{\mathrm{o}}+G_{\mathrm{o}} \frac{v_{\mathrm{i}}}{V_{\mathrm{o}}}+\frac{1+f v_{\mathrm{i}} / V_{\mathrm{o}}}{K}-\sqrt{\left(\mathbf{C D}_{\mathrm{o}}+G_{\mathrm{o}} \frac{v_{\mathrm{i}}}{V_{\mathrm{o}}}+\frac{1+f v_{\mathrm{i}} / V_{\mathrm{o}}}{K}\right)^{2}-4 \mathbf{C D}_{\mathrm{o}} G_{\mathrm{o}} \frac{v_{\mathrm{i}}}{V_{\mathrm{o}}}}\right)}{1+f v_{\mathrm{i}} / V_{\mathrm{o}}}$

The polarimetric method proposed by us undoubtedly shows interesting issues. Considered that the major role in the measurement process is played by the characteristics of the host rather than those of the guest, our method may
$12,300 \mathrm{M}^{-1}$, i.e., over almost 4 orders of magnitude) without any particular difficulty, the only limitation being given by the intrinsic solubility of the guest, according to the relationship 4:

Table 1. Polarimetric data

\begin{tabular}{|c|c|c|c|c|c|c|}
\hline Host & Guest & $\mathrm{pH}$ & $\Delta \Theta\left(\operatorname{deg} \mathrm{dm}^{-1} \mathrm{M}^{-1}\right)$ & $K\left(\mathrm{M}^{-1}\right)$ & $\log K$ & $\log K$ lit. $^{\mathrm{a}}$ \\
\hline \multirow[t]{10}{*}{$\alpha-\mathbf{C D}$} & $1^{\mathrm{c}}$ & 6.0 & $-14.7 \pm 0.3$ & $2630 \pm 490$ & $3.24 \pm 0.08$ & $3.37 \mathrm{cal}, 3.38 \mathrm{cal}$ \\
\hline & $3^{\mathrm{c}}$ & 2.5 & $-8.9 \pm 0.3$ & $169 \pm 23$ & $2.28 \pm 0.06$ & 1.70 pot \\
\hline & $5^{\mathrm{b}}$ & 9.2 & $-17.6 \pm 1.6$ & $53 \pm 8$ & $1.72 \pm 0.07$ & $2.15 \mathrm{cal}, 2.16 \mathrm{cal}, 1.93 \mathrm{cal}$ \\
\hline & $7^{\mathrm{c}}$ & 6.0 & $-18.4 \pm 0.9$ & $3.7 \pm 0.3$ & $0.57 \pm 0.04$ & $4.2 \mathrm{cal}, 1.57 \mathrm{cal}, 1.46 \mathrm{uv}, 1.32 \mathrm{cal}$ \\
\hline & $8^{\mathrm{b}}$ & 2.5 & $+12.7 \pm 0.3$ & $1350 \pm 155$ & $3.13 \pm 0.05$ & $2.88 \mathrm{pot}, 3.0 \mathrm{cal}, 2.52 \mathrm{~cd}, 2.96 \mathrm{cal}, 2.77 \mathrm{cal}$ \\
\hline & $\mathbf{9}^{\mathrm{b}}$ & 2.5 & $+25.9 \pm 0.3$ & $330 \pm 55$ & $2.52 \pm 0.03$ & $2.52 \mathrm{cal}, 2.34 \mathrm{cal} \mathrm{uv}, 2.30 \mathrm{cal}$ uv, $2.25 \mathrm{uv}, 2.31 \mathrm{~cd}, 2.01 \mathrm{cal}$ \\
\hline & $9^{b}$ & 11.0 & $+104.2 \pm 1.8$ & $3310 \pm 460$ & $3.52 \pm 0.06$ & $\begin{array}{l}3.08 \mathrm{cal}, 3.55 \mathrm{uv}, 3.42 \mathrm{uv}, 3.37 \mathrm{cal} \mathrm{uv}, 3.30 \mathrm{cal} \mathrm{uv}, \\
3.26 \mathrm{cal}, 3.19 \mathrm{cal}\end{array}$ \\
\hline & $10^{\mathrm{b}}$ & 6.0 & $+90.2 \pm 1.6$ & $1550 \pm 140$ & $3.19 \pm 0.04$ & $3.02 \mathrm{uv}$ \\
\hline & $11^{\mathrm{c}}$ & 6.0 & $+102.5 \pm 0.9$ & $2755 \pm 190$ & $3.44 \pm 0.03$ & $3.12 \mathrm{uv}$ \\
\hline & $12^{\mathrm{c}}$ & 6.0 & $+102.5 \pm 1.1$ & $1780 \pm 125$ & $3.25 \pm 0.03$ & - \\
\hline \multirow[t]{18}{*}{$\beta-\mathbf{C D}$} & $\mathbf{1}^{\mathrm{c}}$ & 6.0 & $-7.1 \pm 0.3$ & $1045 \pm 215$ & $3.02 \pm 0.09$ & $2.62 \mathrm{cal}$ \\
\hline & $2^{\mathrm{b}}$ & 6.0 & $-11.5 \pm 0.3$ & $660 \pm 60$ & $2.82 \pm 0.04$ & $2.84 \mathrm{cal}, 2.70 \mathrm{uv}, 2.85 \mathrm{cal}$ \\
\hline & $3^{\mathrm{c}}$ & 2.5 & $-8.2 \pm 0.2$ & $5210 \pm 850$ & $3.72 \pm 0.07$ & $3.61 \mathrm{uv}$ \\
\hline & $4^{\mathrm{b}}$ & 11.0 & $-12.7 \pm 0.4$ & $195 \pm 22$ & $2.29 \pm 0.05$ & - \\
\hline & $5^{\mathrm{b}}$ & 9.2 & $-21.9 \pm 0.3$ & $12,300 \pm 1980$ & $4.09 \pm 0.07$ & $4.29 \mathrm{cal}, 4.51 \mathrm{cal}, 4.60 \mathrm{cal}$ \\
\hline & $6^{\mathrm{c}}$ & 6.0 & $-21.0 \pm 0.4$ & $3160 \pm 360$ & $3.50 \pm 0.05$ & 5.04 pot, $3.95 \mathrm{uv}, 3.93 \mathrm{uv}, 3.89 \mathrm{cal}$ \\
\hline & $7^{\mathrm{c}}$ & 6.0 & $-15.0 \pm 1.1$ & $16.2 \pm 0.4$ & $1.21 \pm 0.01$ & $1.97 \mathrm{cal}, 3.4 \mathrm{cal}$ \\
\hline & $8^{\mathrm{b}}$ & 2.5 & $+9.8 \pm 0.3$ & $955 \pm 130$ & $2.98 \pm 0.06$ & $2.1 \mathrm{cal}, 3.26 \mathrm{cal}, 2.74 \mathrm{pot}, 2.52 \mathrm{~cd}$ \\
\hline & $9^{\mathrm{b}}$ & 2.5 & $+13.5 \pm 0.2$ & $740 \pm 70$ & $2.87 \pm 0.04$ & $\begin{array}{l}3.0 \mathrm{cal}, 2.54 \mathrm{cal}, 2.50 \mathrm{cal}, 2.48 \mathrm{uv}, 2.41 \mathrm{cal}, 2.28 \mathrm{~cd} \text {, } \\
1.76 \mathrm{lc}\end{array}$ \\
\hline & $9^{\mathrm{b}}$ & 11.0 & $+81.7 \pm 0.2$ & $813 \pm 19$ & $2.91 \pm 0.01$ & $2.57 \mathrm{cal}, 3.25$ pot, $2.97 \mathrm{uv}, 2.80 \mathrm{uv}, 2.76 \mathrm{cal} \mathrm{uv}, 2.63 \mathrm{cal}$ \\
\hline & $10^{\mathrm{b}}$ & 6.0 & $+74.6 \pm 0.8$ & $603 \pm 14$ & $2.78 \pm 0.01$ & $2.79 \mathrm{uv}$ \\
\hline & $11^{\mathrm{c}}$ & 6.0 & $+79.6 \pm 0.9$ & $1065 \pm 60$ & $3.03 \pm 0.03$ & $2.87 \mathrm{uv}$ \\
\hline & $12^{\mathrm{c}}$ & 6.0 & $+88.9 \pm 0.6$ & $500 \pm 30$ & $2.70 \pm 0.03$ & - \\
\hline & $13^{\mathrm{c}}$ & 6.0 & $+59.8 \pm 1.3$ & $324 \pm 22$ & $2.51 \pm 0.03$ & $2.54 \mathrm{uv}$ \\
\hline & 14- $R^{\mathrm{c}}$ & 6.0 & $+51.9 \pm 1.5$ & $288 \pm 20$ & $2.46 \pm 0.03$ & $2.60 \mathrm{uv}$ \\
\hline & $14-S^{c}$ & 6.0 & $+59.8 \pm 0.7$ & $479 \pm 22$ & $2.68 \pm 0.02$ & $2.73 \mathrm{uv}$ \\
\hline & $15^{\mathrm{c}}$ & 6.0 & $+80.0 \pm 1.0$ & $1040 \pm 60$ & $3.02 \pm 0.03$ & $2.94 \mathrm{uv}$ \\
\hline & $16^{\mathrm{c}}$ & 2.5 & $-7.3 \pm 0.3$ & $238 \pm 33$ & $2.38 \pm 0.06$ & - \\
\hline$\gamma-\mathbf{C D}$ & $5^{\mathrm{c}}$ & 2.5 & $-24.7 \pm 1.0$ & $234 \pm 22$ & $2.37 \pm 0.04$ & $3.52 \mathrm{cal}, 2.44 \mathrm{uv}$ \\
\hline
\end{tabular}

${ }^{a}$ Data from Refs. 7f and 11; cal: calorimetry; pot: potentiometry; uv: spectrophotometry; lc: liquid chromatography.

b From Ref. 18 .

c This work. 
$K \geq\left(\frac{G_{\max }}{3}+\frac{\mathbf{C D}_{\mathbf{o}}}{4}\right)^{-1}$

where $G_{\max }$ is guest solubility. Data reproducibility was in general very satisfactory (within $3 \%$ ).

The behavior of aliphatic guests 1-6, in comparison with phenol $\mathbf{7}$ and benzoic acid 8, clearly evidences how much challenging the rationalization of all the different effects on the binding phenomenon can be. Let us consider first the guests 1 (protonated cationic form at $\mathrm{pH} 6.0$ ), 3 (undissociated neutral form at $\mathrm{pH} 2.5$ ), and 5 (dissociated anionic form at $\mathrm{pH}$ 9.2), for which polarimetric data with both $\alpha-C D$ and $\beta-C D$ are available. These substrates show a dramatic decrease of the binding constant with $\alpha-\mathbf{C D}$ $\left(K_{\alpha, \mathbf{1}}>K_{\alpha, \mathbf{3}}>K_{\alpha, \mathbf{5}}\right)$ on increasing their steric requirements, whereas the opposite behavior is found with $\beta-\mathbf{C D}$ $\left(K_{\beta, \mathbf{1}}<K_{\beta, \mathbf{3}}<K_{\beta, \mathbf{5}}\right)$. This finding agrees with the conclusions obtained by Matsui and Mochida ${ }^{7 \mathrm{a}}$ studying the inclusion of alcohols by means of a QSAR-type approach. Inclusion of $\mathbf{1}$ and 3 in $\alpha-\mathbf{C D}$ leads to larger absolute $\Delta \Theta$ values with respect to $\beta-\mathbf{C D}$, and in general the same behavior is observed also for all the other guests (with the only exception of $\mathbf{5}$, see below). This finding can be easily explained considering that the inclusion in the narrowest host implies stricter conformational constraints and leads to tighter complexes. For aliphatic guests, indeed, having no chromophore active in the UV-vis spectral region, the occurrence of any significant contribution to $\Delta \Theta$ due to induced circular dichroism effects can be reasonably ruled out. Therefore, $\Delta \Theta$ should essentially account for the dynamic conformational changes of the CD macrocycle. It is worth noting that absolute $\Delta \Theta$ values with $\beta-\mathbf{C D}$ tend to increase on increasing the steric requirements of the guests and on decreasing their conformational freedom.

The very large $K$ value found for the binding between $\mathbf{5}$ and $\beta$-CD has been already explained by other authors by the occurrence of particularly favorable host-guest hydrophobic/ van der Waals interactions, due to nearly perfect size fit. ${ }^{24}$ Noticeably, $\mathbf{5}$ and its amine analogue $\mathbf{6}$ show the largest negative $\Delta \Theta$ values found in the present investigation. By contrast, the relatively small $\Delta \Theta$ value for the inclusion of $\mathbf{5}$ in $\alpha-\mathbf{C D}$ (together with the relevant low $K$ value) accounts for the formation of a shallow complex, because of the difficult penetration of the very bulky guest into the narrow host. As an interesting consequence, this is the only case in which a smaller absolute value for $\Delta \Theta$ is found with $\alpha$-CD than with $\beta-C D$. Previous NMR and computational (CHARMm22) results reported by Schneider ${ }^{25}$ are in full agreement with these conclusions. Furthermore, the inclusion of $\mathbf{5}$ in $\gamma$-CD leads to a smaller $K$ value with respect to $\beta$-CD, but also to a larger $\Delta \Theta$ absolute value. The former observation is easily explained admitting the formation of a relatively flexible complex with $\gamma-\mathbf{C D}$, as accounted for by the occurrence of a nearly null inclusion $\Delta H^{\circ}$ value reported by Eftink, ${ }^{26}$ along with a very favorable $T \Delta S^{\circ}$ term. Nevertheless, the increase of $\Delta \Theta$ value actually accounts for the reorganization of a larger network of glucose units: as a matter of fact, the contribution to $\Delta \Theta$ per glucose unit is nearly the same (ca. $3.1 \mathrm{deg} \mathrm{dm}^{-1} \mathrm{M}^{-1}$ ) for both $\beta-\mathbf{C D}$ and $\gamma$-CD.
Interestingly, the inclusion of the amantadine cation $(6$ at $\mathrm{pH}$ 6.0 ) in $\beta$-CD leads to a smaller $K$ value with respect to $\mathbf{5}$, but to nearly the same $\Delta \Theta$ value. This suggests that the steric constraints implied in the formation of both complexes are nearly the same, irrespective of the actual charge of the guest; consequently the occurrence of very similar van der Waals interactions with the host cavity may be presumed. Therefore, differences in complex stabilities should mainly account only for the occurrence of different solvation effects upon the guest. On the other hand, the 1,4-diazabicyclooctane 4 shows an interesting behavior with $\beta$-CD. Although it presents an intermediate steric bulk between the 'large' guests 5 and $\mathbf{6}$ and the 'small' ones 1-3, it shows a quite low $K$ value and a $\Delta \Theta$ value comparable with the small cyclohexanol 2. Computational models (MM2/QD) in the gas phase show that the hydrogen bond acceptor properties of the two nitrogen atoms, together with the lack of an overall dipole moment (see later) due to molecular symmetry, hampers a deep penetration of the guest into the host cavity and forces it to reside near the secondary host rim (Fig. 3). The occurrence of such a shallow arrangement also explains the modest affinity for inclusion.

Before proceeding with the present discussion, it is useful to remember that the $\mathbf{C D}$ cavity possesses a permanent dipole moment, ${ }^{27}$ aligned along the ideal cavity axis and having its positive pole directed toward the primary hydroxyl rim. Its interaction with any occurring dipole moment of the guest plays an important role in determining the energetics of the inclusion processes, in particular with the narrowest $\alpha$ CD. Under this perspective, the comparison of the aliphatic guests cyclohexanol $\mathbf{2}$ and cyclohexanecarboxylic acid $\mathbf{3}$ with their aromatic analogues phenol $\mathbf{7}$ and benzoic acid $\mathbf{8}$ appears quite intriguing. Inclusion in $\beta-\mathbf{C D}$ is strikingly favored for the aliphatic derivatives, whereas the opposite seems true for $\alpha-\mathbf{C D}$ (it is worth noting that we were not able to get a reliable estimate for the very low binding constant between $\mathbf{2}$ and $\alpha-\mathbf{C D})$. This behavior suggests that, in full agreement with the previous discussion, the inner wall of the $\beta$-CD cavity experiences more favorable hydrophobic and van der Waals interactions with the aliphatic cyclohexyl group than with the aromatic phenyl group, according to the trend shown by the values for the relevant steric constants $E_{\mathrm{s}}$ reported in the literature. ${ }^{28}$ Nevertheless, differences in polarizability may also play their own role: as a matter of fact, the inclusion in $\alpha-\mathbf{C D}$ neatly favors the aromatic guest $\mathbf{8}$ with respect to its aliphatic analogue $\mathbf{3}$. Inclusion of the

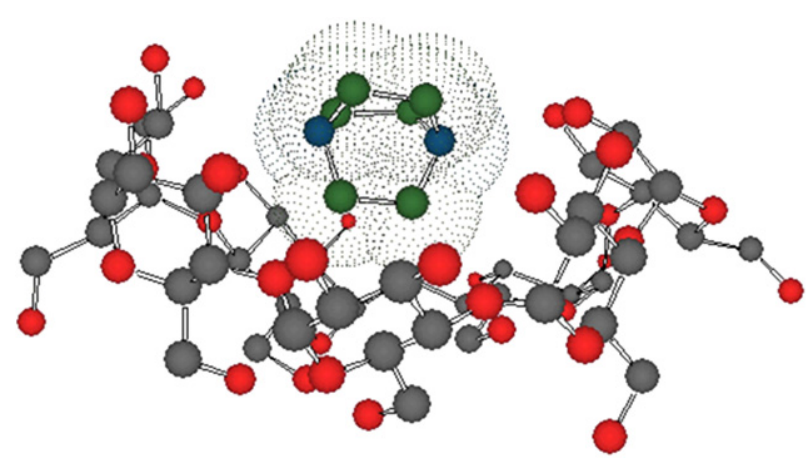

Figure 3. Model (MM2/QD) of the inclusion complex $\beta-\mathbf{C D} \cdot \mathbf{4}$. The guest is evidenced by the dot surface and by the different color shading; hydrogen atoms are not shown for clarity. 
carboxyl derivatives $\mathbf{3}$ and $\mathbf{8}$ is always favored with respect to the corresponding hydroxyl homologues $\mathbf{2}$ and 7, and differences in $\log K$ values are larger for $\alpha-\mathbf{C D}$ than for $\beta-\mathbf{C D}$, once again suggesting the greater importance of polar interactions for the narrowest host.

The discussion of $\Delta \Theta$ for $\mathbf{7}$ and $\mathbf{8}$ is complicated by the presence of the phenyl ring chromophore, which implies the possible occurrence of polarizability/induced dichroism effects on both the absolute value and the sign of this parameter. As will be illustrated more in detail later, positive $\Delta \Theta$ values are detected every time a strongly polarized aryl ring is included. Benzoic acid $\mathbf{8}$ shows a positive $\Delta \Theta$, whereas a negative value is found with phenol 7. It is worth noting that NMR, computational (MM2, AM1) and crystallographic results ${ }^{29}$ all agree in concluding that $\mathbf{8}$ penetrates the $\beta$-CD cavity bearing the functional carboxyl group directed toward the primary hydroxyl rim; by contrast, some computational results ${ }^{16 b, 29 b}$ indicate that the situation for 7 is more complex. Our dynamic (MM2) simulations suggest that this small guest is able to preserve a certain rotational freedom within the cavity, allowing it to assume different orientations with respect to the ideal CD axis; however, it mainly tends to reside near the primary hydroxyl rim and with its $C_{2}$ symmetry axis almost perpendicular to the ideal CD axis.

$p$-Nitrophenol 9 and $p$-nitro-aniline derivatives 10, 11 and 13-15 constitute an important group of guests, the inclusion of which in CDs has been extensively studied from a thermochemical point of view $^{7 \mathrm{~d}, \mathrm{f}, 11}$ (the behavior of diethanolamine derivative $\mathbf{1 2}$ and of the imidazole derivative $\mathbf{1 6}$ has never been studied previously). Their binding equilibria are strongly affected by polar effects, because of the interaction between the dipole moment of the aromatic moiety and the local permanent electric field of the $\mathbf{C D}$ cavity. As a structural consequence, these guests always penetrate into the host cavity with the nitro group unambiguously directed toward the primary host rim. ${ }^{30}$ Polar effects are particularly important for $\boldsymbol{\alpha}-\mathbf{C D}$, which is able to bind tightly only the aromatic moiety of the guest. As a matter of fact, $p$-nitrophenol 9 in its neutral form (at $\mathrm{pH} 2.5$ ) shows a binding constant toward $\alpha$-CD 100 times larger than simple phenol 7. Moreover, 9 further increases its binding constant by exactly 1 order of magnitude on passing to its ionized form (at $\mathrm{pH}$ 11.0). It is interesting to observe at the same time a dramatic increase of the $\Delta \Theta$ value (ca. $78 \mathrm{deg} \mathrm{dm}^{-1} \mathrm{M}^{-1}$ ), showing how strongly this parameter too is affected by the electronic distribution and by the dipolar character of the guest molecule, both for intrinsic reasons ${ }^{20}$ and for the effect on the overall steric constraints induced upon inclusion. Bergeron ${ }^{31}$ claimed the actual occurrence of a strong induced circular dichroism effect on the aromatic chromophore, and stated that this contribution neatly overwhelms the one due to the conformational rearrangement of the host. Nevertheless, the inversion of sign for $\Delta \Theta$ with respect to simple phenol 7 is also noteworthy, having no unambiguously defined direction of penetration into the host cavity (the same is observed for $\beta$-CD too). On passing to $\beta$-CD, factors such as hydrophobic and solvation effects, van der Waals interactions and hydrogen bonding grow in importance with respect to $\alpha-\mathbf{C D}$, owing to the fact that the larger cavity is able to contain the entire guest, ancillary chain included, and still preserves a certain degree of flexibility. ${ }^{11}$ The binding constant for the inclusion of $\mathbf{9}$ in $\beta$-CD is only slightly higher at $\mathrm{pH} 11.0$ than at $\mathrm{pH} 2.5$, probably because of both the high desolvation energy and the unfavorable interaction between the hydrophobic inner cavity wall and the charged guest. Moreover, the difference in $\Delta \Theta$ values between the anionic form and neutral one is significantly lower for $\beta$ CD than for $\alpha$-CD $\left(9.7 \mathrm{deg} \mathrm{dm}^{-1} \mathrm{M}^{-1}\right.$ per glucose unit in the former case, $13.1 \mathrm{deg} \mathrm{dm}^{-1} \mathrm{M}^{-1}$ per glucose unit in the latter one), accounting for the occurrence of less strict conformational constraints.

For $p$-nitroaniline derivatives the binding constants with $\alpha$ CD depend to some extent also on the interaction between the 'ancillary chain' of the guest and the 'expanded hydrophobic sphere', constituted by the structured water cage near the secondary host rim. ${ }^{7 \mathrm{~d}, 11}$ It is interesting to note that the $K$ values for guests 10-12 seem to vary according to the hydrophobic character of their ancillary chains. However, $\Delta \Theta$ values seem rather a function of the substitution pattern on the aniline $\mathrm{N}$ atom. The $N$-mono-substituted derivative 10 shows a $\Delta \Theta$ value lower by ca. $12 \mathrm{deg} \mathrm{dm}^{-1} \mathrm{M}^{-1}$ than both the $N$-di-substituted derivatives 11 and 12. This appears to be the likely consequence of the electron releasing effect of the additional alkyl group on the electronic distribution of the guest.

On passing to $\beta-\mathbf{C D}, \Delta \Theta$ values for derivatives 10-16 show surprisingly large variations, ranging from $+88.9 \mathrm{deg} \mathrm{dm}^{-1} \mathrm{M}^{-1}$ for 12 down to $+51.9 \mathrm{deg} \mathrm{dm}{ }^{-1} \mathrm{M}^{-1}$ for $14-R^{32}$ and even to $-7.3 \mathrm{deg} \mathrm{dm}^{-1} \mathrm{M}^{-1}$ for 16. These results can be only partly explained with the occurrence of purely electronic effects on the guest. As a matter of fact, the difference in $\Delta \Theta$ values between $\mathbf{1 0}$ and $\mathbf{1 1}$ is only $5 \mathrm{deg} \mathrm{dm}^{-1} \mathrm{M}^{-1}$ with $\beta$-CD, whereas $\mathbf{1 1}$ and $\mathbf{1 2}$ now show a difference of

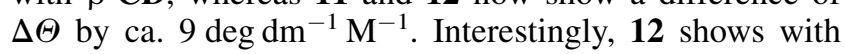
$\beta-\mathbf{C D}$ a slightly lower binding constant than 10, whereas the opposite is found with $\alpha-\mathbf{C D}$. A previous thermodynamic study ${ }^{11}$ showed that the inclusion in $\beta-\mathbf{C D}$ of these $p$-nitroaniline derivatives is basically enthalpy driven for those guests having a hydrophobic (or only moderately hydrophilic) and flexible ancillary chain. On the other hand, for guests having a chain able to form two or more hydrogen bonds, the resulting complex is so rigid that unfavorable entropy contributions prevail and, consequently, on increasing the $-\Delta H^{\mathrm{o}}$ of inclusion the relevant $K$ values decrease. Guests 11 and 15 have been recognized to fall into the former group, whereas 10, 13, and 14- $R /-S$ belong to the latter one. ${ }^{11}$ It is noteworthy that the former two guests show quite similar $\Delta \Theta$ values (ca. $+80 \mathrm{deg} \mathrm{dm}{ }^{-1} \mathrm{M}^{-1}$ ), whereas for the guests of the second group a decrease of $\Delta \Theta$ can be observed (passing from +74.6 to $51.9 \mathrm{deg} \mathrm{dm}^{-1} \mathrm{M}^{-1}$ ) on decreasing the corresponding $K$ value. This behavior is somewhat surprising, because under these circumstances the inclusion complex becomes more and more rigid (as accounted for by the relevant $T \Delta S^{\mathrm{o}}$ values), and therefore an increase of $\Delta \Theta$ should be expected. However, computational (MM2/ QD) models in the gas phase for guest $\mathbf{1 0}$ show that an effective hydrogen bond interaction may occur between the $\mathrm{H}-\mathrm{N}^{\prime}$ aniline group and the glycosidic $\mathrm{O}$ bridge atoms. This interaction allows for the complex the existence of several energy minima in which the guest is forced into the $\beta$ CD cavity in such a way that its dipole momentum cannot 

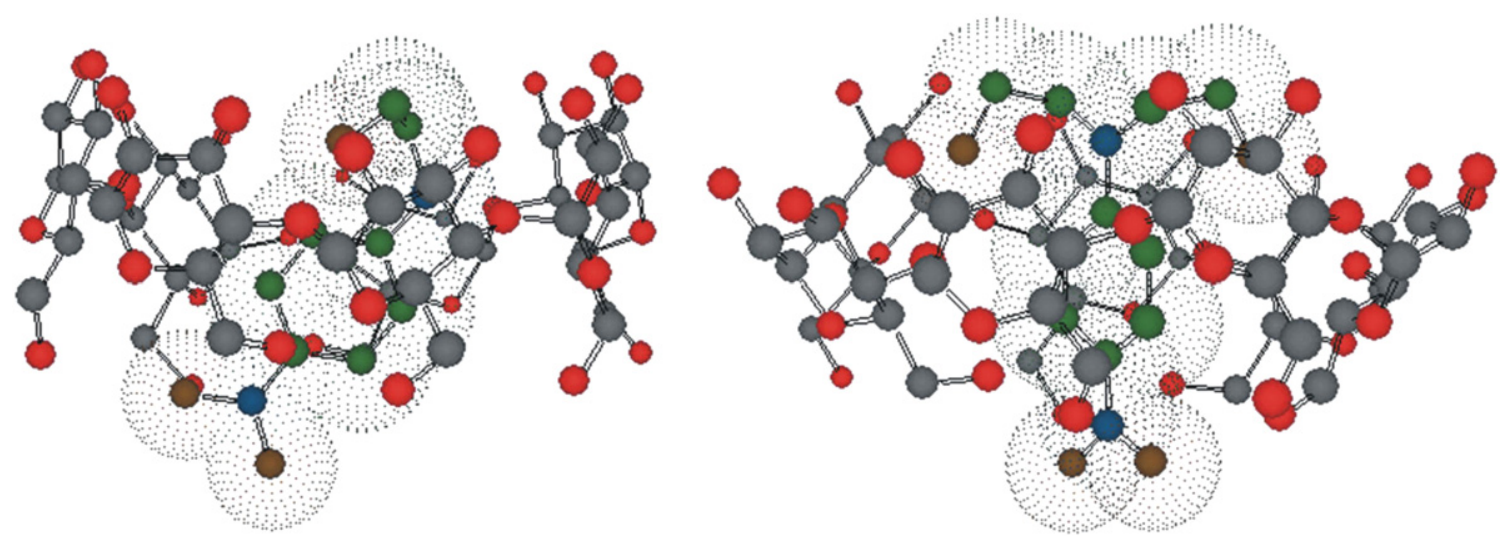

Figure 4. Models $(\mathrm{MM} / \mathrm{QD})$ of the inclusion complexes $\beta-\mathbf{C D} \cdot \mathbf{1 0}$ (left) and $\beta-\mathbf{C D} \cdot \mathbf{1 2}$ (right). The guests are evidenced by the dot surfaces and by the different color shading; hydrogen atoms are not shown for clarity.

be any longer aligned with the ideal cavity axis (Fig. 4 left). Consequently, induced circular dichroism effects (or any other electronic effect) on $\Delta \Theta$ become less effective, as experimentally observed.

The behavior of the diethanolamine derivative $\mathbf{1 2}$ is quite interesting. As a matter of fact, its ancillary chain is able to form two hydrogen bonds and, according to the previous discussion, its inclusion should be entropically disfavored with respect to sarcosole derivative 11, as accounted for by the observed $K$ values. However, the lack of a free $\mathrm{H}-\mathrm{N}^{\prime}$ group, as well as its overall symmetry, probably forces $\mathbf{1 2}$ to reside in axis with respect to the $\beta$-CD cavity (Fig. 4 right). Therefore, electronic effects on $\Delta \Theta$ are fully operating and we are able to record the largest $\Delta \Theta$ value $\left(+88.9 \mathrm{deg} \mathrm{dm}^{-1} \mathrm{M}^{-1}\right)$ observed with $\beta$-CD. It is noteworthy that this value is anyhow lower by ca. $13 \mathrm{deg} \mathrm{dm}{ }^{-1} \mathrm{M}^{-1}$ than that observed with $\boldsymbol{\alpha}$-CD. The imidazolium derivative 16 (at $\mathrm{pH} 2.5$ ) offers another interesting case. As a matter of fact, owing to the electric charge and the peculiar electronic demand of the imidazolium ancillary chain, this guest is moderately hydrophilic, and its $p$-nitrophenyl moiety has a poor dipole character. Consequently, its binding constant is low and, more remarkably, it shows a negative $\Delta \Theta$ value, unlike all the other members of the group. In fact, $\mathbf{1 6}$ cannot really be considered homogeneous with guests 10-15 discussed above.

\section{Conclusions}

The present study confirms polarimetry to be a suitable, versatile and reliable tool for the evaluation of the binding constants between cyclodextrins and various organic guest molecules. Interesting microscopic information can be inferred from the analysis of polarimetric data, which can be profitably compared with thermodynamic data. In particular, the trends shown by the $\Delta \Theta$ values, although depending on both conformational and induced dichroism effects, may constitute a suitable mean to evaluate the extent of conformational changes or constraints associated to the formation of the inclusion complex. This information is 'directly' accessible for aliphatic guests, but can be reasonably achieved whenever we carefully examine the behavior of strictly homogeneous aromatic guests, even if heavy polarity effects occur.
Finally, a few words should be made about the very interesting comparison between the $K$ values reported here and the relevant literature data for the same host-guest couples. It is noteworthy that, in several occasions, the binding constant estimate for the same host-guest couple by means of different methodologies (or even using the same methodology by different authors) afforded strikingly different results. ${ }^{7 \mathrm{f}}$ For instance, $K$ values ranging from 128 to $1820 \mathrm{M}^{-1}$ have been found for the binding constant between benzoic acid 8 and $\beta-\mathbf{C D}$. Different calorimetric measurements for the inclusion of phenol 7 in both $\alpha-\mathbf{C D}$ and $\beta-\mathbf{C D}$ afforded results differing by even more than 1 order of magnitude. ${ }^{7 \mathrm{f}}$ Similar situations occur for $p$-nitrophenol 9 and its anion and, to a lesser extent, also for adamantane derivatives $\mathbf{5}$ and $\mathbf{6}$. Our results show fair to excellent agreement with previous reports in half of the cases examined. In particular, the best agreement has been found with data obtained by means of UV-vis spectrophotometric titrations. Among the hostguest couples for which $K$ values spread over a wide range have been previously reported (in particular with guests $\mathbf{5}$ 9), in four cases our datum falls within the range and appears as a good compromise between the other different values, whereas in two other cases our datum is just a little larger than the maximum value of the range. By contrast, for the inclusion of adamantane derivatives $\mathbf{5}$ and $\mathbf{6}$ and of phenol 7 our results are much lower than previous reports. In the case of 7, in particular, it is worth mentioning that we performed our experiments in systems at higher guest concentrations (up to ca. $0.24 \mathrm{M}$ ) than those reported for previous calorimetric determinations. Therefore, our results benefit from observations carried out on systems where the binding equilibrium had been forced toward complex formation up to a higher extent. ${ }^{33}$ Anyway, all the previous considerations on the whole account for a good reliability of our method, as well as of the reasonability of the assumptions made. ${ }^{20}$

\section{Experimental section}

\subsection{Materials}

All commercial reagents and materials needed were used as purchased, with no further purification. Phosphate $(\mathrm{pH} 2.5$, 6.0 and 11.0) and ammonia (pH 9.2) buffer stock solutions $(0.05 \mathrm{M})$ in double-distilled water were prepared and used as solvents for the preparation of measurement solutions; 
actual buffer $\mathrm{pH}$ values were checked before use by means of a routine $\mathrm{pH}$-meter equipment. Compounds 10, 11 and 13-15 were prepared, purified and characterized as already described elsewhere. ${ }^{11}$

\section{2. $N$-(4-Nitrophenyl)-diethanolamine 12}

To a solution of diethanolamine $(1.05 \mathrm{~g}, 10 \mathrm{mmol})$ in DMSO $(15 \mathrm{~mL})$ a slight excess of 4-nitro-fluorobenzene $(1.48 \mathrm{~g}$, $10.5 \mathrm{mmol})$ and solid dry $\mathrm{K}_{2} \mathrm{CO}_{3}(1.52 \mathrm{~g}, 11 \mathrm{mmol})$ were added. The mixture was kept at $60^{\circ} \mathrm{C}$ overnight under stirring. Then the crude product was poured into water $(200 \mathrm{~mL})$ and extracted with four portions of ethyl acetate $(60 \mathrm{~mL}$ each). The joined organic extracts were dried on sodium sulfate and vacuum distilled. The residue was crystallized from ethanol-light petrol (yield $1.81 \mathrm{~g}, 80 \%$ ). Mp 105-106 ${ }^{\circ} \mathrm{C} .{ }^{1} \mathrm{H}$ NMR (DMSO- $d_{6}, 300 \mathrm{MHz}$ ): $\delta 3.52-3.67$ $(\mathrm{m}, 8 \mathrm{H}), 4.89(\mathrm{t}, J=5.1 \mathrm{~Hz}, 2 \mathrm{H}), 6.79$ and $7.99(2 \mathrm{~d}, J=$ $9.6 \mathrm{~Hz}$, each $2 \mathrm{H}) .{ }^{13} \mathrm{C}$ NMR (DMSO-d $6,300 \mathrm{MHz}$ ): $\delta$ 53.3, 58.1, 110.7, 125.9, 135.34, 153.4. Anal. Calcd for $\mathrm{C}_{10} \mathrm{H}_{14} \mathrm{~N}_{2} \mathrm{O}_{4}$ : C, 53.09; $\mathrm{H}, 6.24 ; \mathrm{N}$, 12.38. Found: C, 53.18; H, 6.29; N, 12.30 .

\subsection{1-(4-Nitrophenyl)-imidazole 16}

Imidazole $(0.68 \mathrm{~g}, 10 \mathrm{mmol})$ and $\mathrm{KOH}(0.56 \mathrm{~g}, 10 \mathrm{mmol})$ were dissolved in methanol $(25 \mathrm{~mL})$; the solution was stirred for a few minutes and then vacuum distilled. The residue was dissolved in DMSO $(15 \mathrm{~mL})$, and to the solution a slight excess of 4-nitro-fluorobenzene $(1.48 \mathrm{~g}, 10.5 \mathrm{mmol})$ was added. The mixture was kept overnight at $60{ }^{\circ} \mathrm{C}$ under stirring. Then the crude product was poured into water $(200 \mathrm{~mL})$ and filtered off. The residue was crystallized from methanol/water (yield $1.32 \mathrm{~g}, 70 \%$ ). Mp 201-202 ${ }^{\circ} \mathrm{C}$. ${ }^{1} \mathrm{H}$ NMR (DMSO- $\left.d_{6}, 300 \mathrm{MHz}\right): \delta 7.17$ (s, 1H), $7.91(\mathrm{~s}$, $1 \mathrm{H}), 7.93$ and $8.30(2 \mathrm{~d}, J=9.0 \mathrm{~Hz}$, each $2 \mathrm{H}), 8.47$ (s, 1H). ${ }^{13} \mathrm{C}$ NMR (DMSO- $\left.d_{6}, 300 \mathrm{MHz}\right): \delta 118.0,120.4,125.6$, 131.0, 136.2, 141.8, 145.4. Anal. Calcd for $\mathrm{C}_{9} \mathrm{H}_{7} \mathrm{~N}_{3} \mathrm{O}_{2}$ : C, 57.14; H, 3.73; N, 22.21. Found: C, 57.05; H, 3.79; N, 22.16.

\subsection{Polarimetric measurements}

Polarimetric determinations were performed by means of a JASCO P-1010 polarimeter. Samples for each polarimetric measure experiment were prepared by mixing $5 \mathrm{~mL}$ of a stock CD solution (1.5-7.5 $\mathrm{mM}$ ), dissolved in the proper aqueous buffer, with variable amounts (up to $0.2 \mathrm{~mL}$ ) of solution of the guest in methanol, at least 100 times more concentrated than the $\mathbf{C D}$ solution. Then the optical rotations of the samples were determined as mean values on at least 20 scans, in such a way to achieve an indetermination below 0.0005 deg. Finally, experimental data were subjected to fitting by regression analysis according to Eq. 2 or 3 .

\subsection{MM2/QD computational models}

Models of the inclusion complexes in the gas phase were elaborated by the MM2/QD ${ }^{34}$ method. Molecular mechanics/dynamics computations were performed by means of the CS Chem3D Pro ${ }^{\circledR}$ software using the MM2 force field. For each model a suitable 'simulation pool' was elaborated simulating the dynamic equilibration of the structure at $300 \mathrm{~K}$ for $1000 \mathrm{ps}$ (step $2 \mathrm{fs}$, heating rate $1.0 \mathrm{kcal} / \mathrm{atom} / \mathrm{ps}$ ), in order to explore a significant portion of the potential energy surface of the system. A set of structures was randomly extracted from the 'simulation pool' and the structures were allowed to undergo full energy optimization by means of a 'simulated annealing' procedure (step $1 \mathrm{fs}$, heating rate $0.2 \mathrm{kcal} / \mathrm{atom} / \mathrm{ps}$ ). In this way only a limited number of real energy minima were individuated. Anyway, calculations were not intended for use in quantitative predictions, but only for qualitative comparison purposes.

\section{Acknowledgements}

Italian M.U.R (Rome), PRIN 2006, and University of Palermo (funds for selected research topics) are gratefully acknowledged for financial support.

\section{Supplementary data}

The mathematical derivation and a brief discussion of Eqs. 2 and 3 is reported. Supplementary data associated with this article can be found in the online version, at doi:10.1016/ j.tet.2007.06.065.

\section{References and notes}

1. (a) Szejtli, J. Chem. Rev. 1998, 98, 1743-1753 and references therein; (b) Easton, C. J.; Lincoln, S. F. Chem. Soc. Rev. 1996, 25, 163-170; (c) Szejtli, J.; Osa, T. Comprehensive Supramolecular Chemistry; Atwood, J. L., Davies, J. E. D., MacNicol, D. D., Vögtle, F., Eds.; Pergamon: Oxford, 1996; Vol. 3; (d) Connors, K. A. Chem. Rev. 1997, 97, 1325-1357; (e) Wenz, G. Angew. Chem., Int. Ed. Engl. 1994, 33, 803-822.

2. Guernelli, S.; Laganà, M. F.; Spinelli, D.; Lo Meo, P.; Noto, R.; Riela, S. J. Org. Chem. 2002, 67, 2948-2953.

3. (a) Breslow, R.; Czarnik, A. W.; Lauer, M.; Leppkes, R.; Winkler, J.; Zimmermann, S. J. J. Am. Chem. Soc. 1986, 108, 1969-1979; (b) Breslow, R.; Dong, S. D. Chem. Rev. 1998, 98, 1997-2011; (c) Breslow, R. Acc. Chem. Res. 1995, 28, 146-153.

4. (a) Li, S.; Purdy, W. C.; Schneiderman, E. Chem. Rev. 1992, 92, 1457-1470; (b) Stalcup, A. M. J. Chromatogr., B 2000, 745, 83-102.

5. (a) Ukeama, K.; Hirayama, F.; Irie, T. Chem. Rev. 1998, 98, 2045-2076; (b) Hedges, A. R. Chem. Rev. 1998, 98, 20352044.

6. The amount of papers published on cyclodextrins is so enormous, and concerns so many research fields, that even reviews are virtually countless. Along with the other references reported within the present notes, for a few recent examples see also: (a) See Ref. 1b; (b) Kano, K.; Hasegawa, H. J. Incl. Phenom. Macro. Chem. 2001, 41, 41-47; (c) Dodziuk, H.; Koźmiński, W.; Ejchart, A. Chirality 2004, 16, 90-105; (d) Bicchi, C.; D’Amato, A.; Rubiolo, P. J. Chromatogr., A 1999, 843, 99-121; (e) Fanali, S. J. Chromatogr., A 2000, 875, 89122; (f) Koppenhoefer, B.; Zhu, X.; Jakob, A.; Wuerthner, S.; Lin, B. J. Chromatogr., A 2000, 875, 135-161; (g) Evans, C. E.; Stalcup, A. M. Chirality 2003, 15, 709-723; (h) de Boer, T.; de Zeeuw, R. A.; de Jong, G. J.; Ensing, K. Electrophoresis 2000, 21, 3220-3239; (i) Lipkowitz, K. B. Chem. Rev. 1998, 98, 1829-1873; (j) Lipkowitz, K. B. 
J. Chromatogr., A 2001, 906, 417-442; (k) D’Anna, F.; Lo Meo, P.; Noto, R.; Riela, S. Targets in Heterocyclic Systems; Attanasi, O. A., Spinelli, D., Eds.; S.C.I.: Rome, 2005; Vol. 9, pp 1-38.

7. (a) Matsui, Y.; Mochida, K. Bull. Chem. Soc. Jpn. 1979, 52, 2808-2814; (b) Rekharsky, M. V.; Mayhew, M. P.; Goldberg, R. N.; Ross, P. D.; Yamashoji, Y.; Inoue, Y. J. Phys. Chem. B 1997, 101, 87-100; (c) Wedig, M.; Laug, S.; Christians, T.; Thunhorst, M.; Holzgrabe, U. J. Pharm. Biomed. Anal. 2000, 27, 531-540; (d) Lo Meo, P.; D’Anna, F.; Riela, S.; Gruttadauria, M.; Noto, R. Org. Biomol. Chem. 2003, 1, 1584-1590 and references cited therein; (e) Tabushi, I.; Kiyosuke, Y.; Sugimoto, T.; Yamamura, K. J. Am. Chem. Soc. 1978, 100, 916-919; (f) Rekharsky, M. V.; Inoue, Y. Chem. Rev. 1998, 98, 1875-1917 and references cited therein.

8. (a) Inoue, Y.; Hakushi, T. J. Chem. Soc., Perkin Trans. 2 1985, 935-946; (b) Inoue, Y.; Liu, Y.; Tong, L.-H.; Jin, D.-S. J. Am. Chem. Soc. 1993, 115, 10637-10644; (c) Inoue, Y.; Hakushi, T.; Liu, Y.; Tong, L.-H.; Shen, B.-J.; Jin, D.-S. J. Am. Chem. Soc. 1993, 115, 475-481; (d) Liu, Y.; Han, B.-H.; Li, B.; Zhang, Y.-M.; Zhao, P.; Chen, Y.-T.; Wada, T.; Inoue, Y. J. Org. Chem. 1998, 63, 1444-1454; (e) Rekharsky, M. V.; Inoue, Y. J. Am. Chem. Soc. 2001, 123, 813-826.

9. Rekharsky, M. V.; Inoue, Y. J. Am. Chem. Soc. 2000, 122, 4418-4435.

10. (a) Liu, L.; Guo, Q.-X. Chem. Rev. 2001, 101, 673-695 and references therein; (b) Grunwald, E.; Steel, C. J. Am. Chem. Soc. 1995, 117, 5687-5692; (c) Sharp, K. Protein Sci. 2001, 10, 661-667; (d) Ranatunga, R.; Vitha, M. F.; Carr, P. W. J. Chromatogr., A 2002, 946, 47-49; (e) Vitha, M. F.; Carr, P. W. Ind. Eng. Chem. Res. 2003, 42, 6290-6293.

11. Lo Meo, P.; D’Anna, F.; Gruttadauria, M.; Riela, S.; Noto, R. Tetrahedron 2004, 60, 9099-9111.

12. Yamamura, H.; Rekharsky, M. V.; Ishihara, Y.; Kawai, M.; Inoue, Y. J. Am. Chem. Soc. 2004, 126, 14224-14233.

13. (a) Li, J.; Waldron, K. C. Electrophoresis 1999, 20, 171-179; (b) Rekharsky, M. V.; Inoue, Y. J. Am. Chem. Soc. 2002, 124, 813-826.

14. (a) Corradini, R.; Dossena, A.; Galaverna, G.; Marchelli, R.; Panagia, A.; Sartor, S. J. Org. Chem. 1997, 62, 6283-6289; (b) Pagliari, S.; Corradini, R.; Galaverna, G.; Sforza, S.; Dossena, A.; Montalti, M.; Prodi, L.; Zaccheroni, N.; Marchelli, R. Chem.-Eur. J. 2004, 10, 2749-2758; (c) D’Anna, F.; Lo Meo, P.; Gruttadauria, M.; Riela, S.; Noto, R. Tetrahedron: Asymmetry 2002, 13, 1755-1760; (d) D'Anna, F.; Riela, S.; Gruttadauria, M.; Lo Meo, P.; Noto, R. Tetrahedron 2005, 61, 4577-4583.

15. Rekharsky, M. V.; Yamamura, H.; Kawai, M.; Inoue, I. J. Org. Chem. 2003, 68, 5228-5235.

16. (a) Dodziuk, H. J. Mol. Struct. 2002, 614, 33-45; (b) Mayer, B.; Marconi, G.; Klein, C.; Kohler, G.; Wolschann, P. J. Inclusion Phenom. Mol. Recognit. Chem. 1997, 29, 79-97 and references therein.

17. Connors, K. A. Comprehensive Supramolecular Chemistry; Szejtli, J., Osa, T., Eds.; Pergamon: New York, NY, 1996; Vol. 3, pp 205-240.

18. Lo Meo, P.; D’Anna, F.; Riela, S.; Gruttadauria, M.; Noto, R. Tetrahedron Lett. 2006, 47, 9099-9102.

19. Rees, D. A. J. Chem. Soc. B 1970, 877-884.

20. (a) Kodaka, M. J. Am. Chem. Soc. 1993, 115, 3702-3705; (b) Kodaka, M. J. Phys. Chem. A 1998, 102, 8101-8103; (c) Park, J. W.; Lee, S. Y.; Song, H. J.; Park, K. K. J. Org. Chem. 2005, 70, 9505-9513.
21. Actually, there are literature indications that the presence of methanol may significantly affect the binding constant for some large hydrophobic guests. (See for instance: Mrozek, J.; Guzov, K.; Szabelsky, M.; Karolkzac, J.; Wiczk, W. J. Photochem. Photobiol., A 2002, 153, 121-128; See also: Penn, S. G.; Bergström, E. T.; Goodall, D. M. Anal. Chem. 1994, 66, 2866-2873), whereas this effect seems more limited for more hydrophilic guests (See for instance: Szökő, E.; Gyimesi, J.; Barcza, L.; Magyar, K. J. Chromatogr., A 1996, 754, 181-187). However, some numeric simulations showed us that even for variations in the binding constant up to $20 \%$ consequent to the amount of methanol progressively added, the systematic errors introduced on the best estimations for $K$ and $\Delta \Theta$ are below the limit of the statistic indeterminations inherent to the fitting data procedure. Anyway, the general satisfactory agreement between our data and previous literature reports (see Section 3) accounts for the overall reasonability of our assumptions. Moreover, we actually verified in a blank experiment that small amounts of methanol (up to $4 \% \mathrm{v} / \mathrm{v}$ ) have no significant effect on the molar optical activity of the free $\beta-C D$.

22. The value of the coefficient $f$ for any solvent can be easily estimated on the basis of the density data reported for the proper water-solvent mixtures. For methanol $f=0.938$ (see Ref. 11).

23. Unlike the usual practice, we dropped the common use of specific optical rotations $[\alpha]$, and replaced them with molar optical rotations $[\Theta]$ (measured in $\operatorname{deg} \mathrm{dm}^{-1} \mathrm{M}^{-1}$ ), defined as the optical rotation ideally expected for a sample at a $1 \mathrm{M}$ concentration, under all other usual conditions. This choice agrees with an old suggestion by Rees (see Ref. 19) and simplifies the mathematical treatment, allowing a rational data analysis. To underline this different choice, we also adopted for consistency the symbol $\vartheta$ to denote the observed optical rotations of the samples.

24. (a) Gelb, R. I.; Schwartz, L. M.; Laufer, D. A. J. Chem. Soc., Perkin Trans. 2 1984, 15-21; (b) Eftink, M. R.; Andy, M. L.; Bystrom, K.; Perlmutter, H. D.; Kristol, D. S. J. Am. Chem. Soc. 1989, 111, 6765-6772.

25. Rüdiger, V.; Eliseev, A.; Simova, S.; Schneider, H.-J.; Blandamer, M. J.; Cullis, P. M.; Meyer, A. J. J. Chem. Soc., Perkin Trans. 2 1996, 2119-2122.

26. Cromwell, W. C.; Byström, K.; Eftink, M. R. J. Phys. Chem. 1985, 89, 326-332.

27. (a) Lichtentahler, F. W.; Immel, S. Liebigs Ann. Chem. 1996, 27-37; (b) Sakurai, M.; Kitagawa, M.; Hoshi, H.; Inoue, Y.; Chûjô, R. Chem. Lett. 1998, 895-898.

28. $E_{s}\left(\mathrm{C}_{6} \mathrm{H}_{5}^{-}\right)=-0.38 ; E_{\mathrm{s}}\left(\right.$ cyclo- $\left.\mathrm{C}_{6} \mathrm{H}_{11}^{-}\right)=-0.71$; from Isaacs, N. S. Physical Organic Chemistry; Longman Scientific and Technical Ed.: UK, 1987.

29. (a) Salvatierra, D.; Jaime, C.; Virgili, A.; Sánchez-Ferrando, F. J. Org. Chem. 1996, 61, 9578-9581; (b) Huang, M.-J.; Watts, J. D.; Bodor, N. Int. J. Quantum Chem. 1997, 65, 11351152; (c) Aree, T.; Chaichit, N. Carbohydr. Res. 2003, 338, 439-446.

30. (a) Lü, T.-X.; Zhang, D.-B.; Dong, S.-J. J. Chem. Soc., Faraday Trans. 1989, 85, 1439-1445; (b) Schneider, H.-J.; Hacket, F.; Rüdiger, V.; Ikeda, H. Chem. Rev. 1998, 98, 1755-1785; (c) Bonora, G. M.; Fornasier, R.; Scrimin, P.; Tonellato, U. J. Chem. Soc., Perkin Trans. 2 1985, 367-369; (d) Tee, O. S.; Mazza, C.; Du, X.-X. J. Org. Chem. 1990, 55, 3603-3609.

31. Bergeron, R. J.; Almy Channing, M.; Gibeily, G. J.; Pillor, D. M. J. Am. Chem. Soc. 1977, 99, 5146-5151. 
32. It is worth stressing just here that the polarimetric method is not precluded to chiral guests! Eq. 2 clearly provides the possibility that the guest possesses its own optical activity. So, we included substrates $14-R /-S$ namely to show that our methodology could be profitably applied in such cases too. In other words, the possible contribution to the optical activity of the measurement samples due to the guest can be kept into account upon data fitting, and consequently it does not affect the quality of the eventual results.

33. By the way, at the best of our knowledge no hints in previous literature suggest the occurrence of complexes having a 'non-1:1' stoichiometry between $\beta$-CD and our guests, whereas 2:1 complexes have detected between $\alpha$-CD and some $p$-nitroaniline derivatives at very high host concentration (see for instance Ref. 7d). Nevertheless, for all the cases examined in the present work, the fact that experimental data are very satisfactorily fitted by Eq. 2 or 3, reasonably allows per se to rule out the occurrence of any complex not having the presumed 1:1 stoichiometry.

34. (a) See Ref. 6i; (b) Kozar, T.; Venanzi, C. A. J. Mol. Struct. 1997, 395-396, 451-468. 